Communications in Physics, Vol.20, No.1 (2010), pp. 1-8

\title{
RETRIEVING MOLECULAR STRUCTURAL INFORMATION AND TRACKING HNC/HCN ISOMERIZATION PROCESS WITH HIGH HARMONIC GENERATION BY ULTRASHORT LASER PULSES
}

\author{
NGUYEN NGOC TY AND LE VAN HOANG \\ Department of Physics, \\ Ho Chi Minh City University of Pedagogy \\ VU NGOC TUOC \\ Institute of Engineering Physics, \\ Hanoi University of Technology \\ LE ANH THU \\ J. R. MacDonald Laboratory, Department of Physics, Cardwell Hall, \\ Kansas State University, Manhattan, Kansas 66506, USA
}

\begin{abstract}
We investigate the possibility of applying the iterative method, suggested in our previous work, for HCN molecule and its HNC isomer. We found that the high-order harmonic generation ( $H H G$ ) spectra are quite insensitive to the change of $H-C$ (or $H-N$ ) bond length so that only the inter-nuclear $C-N$ distance can be retrieved from the high-order harmonic spectra using ultrashort intense lasers. Furthermore, by analyzing the HHG spectra emitted by HCN during the chemical reaction path of isomerization we identify the intensity peaks nearby the stable, metastable and transition states. This finding can be useful for tracking the $H N C / H C N$ isomerization process.
\end{abstract}

\section{INTRODUCTION}

With development of the present-day laser technology, femtosecond intense lasers make it possible to observe atoms, molecules in time scale of chemical reaction $[1,2]$. When exposing an atom or a molecule to the intense laser pulse, one of nonlinear optical phenomena occurring is the high-order harmonic generation (HHG) [3]. Because the recombination occurs when the returning electrons are near the target ion, the HHG spectra thus contain information on the structure of the target [4 - 5]. Recently, reconstruction of dynamic molecular structure information from the HHG spectra has involved great interest [6 - 12], especially after Itatani et al reported that they had successfully reconstructed the highest occupied molecular orbital (HOMO) of $\mathrm{N}_{2}$ molecule from the experimental HHG using the tomographic procedure [10].

In [11], theoretical investigation of the tomographic procedure applied for $\mathrm{N}_{2}$ and $\mathrm{O}_{2}$ leads to a conclusion that even under the most favorable conditions, the result from tomographic imaging is still restricted due to the limitation of the HHG plateau region. However, one can obtain the symmetry of the HOMOs and the inter-atomic distances accurately, especially when using lasers of longer wavelengths to generate the HHG. In the 
recent paper [12], we suggested an alternative method to retrieve structural information from the experimental HHG by fitting them with a set of HHG data, theoretically calculated with the given molecular structures. That iterative method has been shown to work quite well for linear molecules such as $\mathrm{N}_{2}, \mathrm{O}_{2}$ and $\mathrm{CO}_{2}$.

In order to have the iterative method to be more practical, the Lewenstein model [13] of HHG calculations need to be improved. A step in this direction was done in [14] by using the scattering waves instead of the plane waves. We note that the developed iterative method has been used also by fitting the laser-induced photoelectron instead of the HHG to obtain the molecular structural information [15].

Thus, a necessary work is to develop the iterative method further to be applicable for molecules that are more complex. In this paper, we will apply this method for the molecule with two structural parameters. The specific linear molecules we are focusing on are hydrogen cyanide and hydrogen isocyanide, which contain two inter-atomic distances, $\mathrm{C}-\mathrm{N}$ and $\mathrm{H}-\mathrm{C}$ ( or $\mathrm{H}-\mathrm{N}$ ). We will illustrate how to apply the iterative method for HCN (HNC) to obtain the structural information from the HHG data.

Isomerization of hydrogen cyanide $(\mathrm{HCN})$ and hydrogen isocyanide $(\mathrm{HNC})$ has attracted great attention [16 - 19]. So far, most of the investigations have been devoted to the temperature dependence of isomerization and the transfer probability due to the collision with inert gas atoms, Ar for instance. In addition, it is worth mentioning [20, 21], in which the authors used ultrashort lasers to stimulate and control HCN/HNC isomerization. The results reported in [16-19] show that the $\mathrm{HCN} / \mathrm{HNC}$ isomerization process is attributed to the classical motion of the hydrogen atom when it receives enough energy to pass the potential barrier and the tunneling effect can be neglected during this process. Thus, we simulate the classical path of the molecule during isomerization process using Born-Oppenheimer approximation, and then calculate HHG spectra with corresponding positions on the classical orbit with different molecular alignments. We have found the HHG intensity peaks at positions to the meta-stable, stable and transition states that allow us to identify the isomers and transition state.

\section{COMPUTATION METHODS}

\section{II.1. Calculation of HHG using Lewenstein model}

Throughout this paper, in order to generate the HHG, a 800nm laser with peak intensity of $2 \times 10^{14} \mathrm{~W} / \mathrm{cm}^{2}$ and duration of $10 \mathrm{fs}$ will be used with the depletion being neglected. We use the Lewenstein model to calculate the theoretical HHG spectra with fixed alignments. The concrete formulas of calculation are available in our previous paper [11], in which, within the single-active-electron approximation, we take the highest occupied molecular orbital for the ground state. The HOMO was obtained from the GAUSSIAN code [22] applying the DFT method with $6-311+\mathrm{G}(2 \mathrm{df}, 2 \mathrm{pd})$ basis set. The continuum state is approximated by a plan wave. The use of the plane wave approximation is probably sufficient for our purpose, as only qualitative features are important. We will need to use the improved Lewenstein model [14] (the so-called quantitative rescattering theory) if more quantitative result is needed. 
The quasi-classical action at the stationary points for the electron propagating in the laser field has a significant contribution in the HHG calculations. This term contains the ionization potential $I_{p}$ of HCN. Therefore, instead of using $I_{p}$ at the equilibrium state of a molecule we will calculate it for each given structural parameters. This can also be calculated conveniently by using the GAUSSIAN code.

Since experimental HHG spectra for HCN are not readily available, in this paper we generate our 'experimental' data from theoretical ones by introducing random errors of the data on each harmonic order of up to $60 \%$, to simulate random 'experimental' uncertainty (see, [12]).

\section{II.2. Molecular dynamics simulation}

We use the position of $\mathrm{H}$ atom described by $\mathrm{R}$ and structural angle $\theta_{H}$ to characterize the chemical reaction path (CRP) of isomerization on the potential energy surface (PES). $\mathrm{R}$ is the distance of $\mathrm{H}$ atom to the center of mass of $\mathrm{C}-\mathrm{N}$. While $\mathrm{H}$ atom is moving on CRP, the other coordinates of $\mathrm{C}$ and $\mathrm{N}$ atoms are optimized with respect to energy. It turns out that the $\mathrm{C}-\mathrm{N}$ distance just lightly oscillates around an equilibrium position. PES is obtained by applying the DFT method with B3LYP/6-311+G(2df,2pd) level theory. It is worth noticing that the accuracy of the B3LYP-level potential energy surface was systematically confirmed from the comparison to experimental measurement [23]. In order to simulate the classical CRP of molecule with given rotational temperature, the number modes of energy absorption and the initial velocity of $\mathrm{H}$ atom we employ the BOMD (BornOppenheimer molecular dynamics) method implemented in the GAUSSIAN 03 package. This method uses a fifth-order polynomial fitted to the energy, gradient, and Hessian at each time step, and then the step size is taken to be much larger than the step size used in the normal method employing only the gradient information [24]. In this calculation, we neglect the energy loss during the isomerization process.

\section{RESULTS AND DISCUSSION}

\section{III.1. Extracting the structural information from HHG spectra}

We repeat our calculation in [11] for the dependence of HHG intensity on molecular alignments. The results in Fig. 1 show that they can serve as an evidence to distinguish the HCN isomers (hydrogen cyanide, hydrogen isocyanide and transition state). Suppose we have already known whether the isomer HCN or HNC in the system. We will use the iterative method to get internuclear separations of a molecule from HHG spectra. The main idea of the iterative method [12] is to compare the experimental HHG spectra with the theoretical data calculated with different structural parameters around the equilibrium position of a molecule. The criterion is to minimize the fitting function and the most suitable theoretical HHG with given molecular structure to the experimental data will be chosen.

We will check sensitiveness of the HHG to structural parameters to make sure whether the iterative method is usable for HCN (HNC). Fig. 2a shows the HHG spectra of $\mathrm{HCN}$ molecule with a fixed internuclear distance $\mathrm{H}-\mathrm{C}$ but distance $\mathrm{C}-\mathrm{N}$ changes by $10 \%$ of length around the equilibrium position. For comparison, we show in Fig. $2 \mathrm{~b}$ the isotropic HHG data corresponding to the distance at stable state but 'experimental' 
errors may get peak at $60 \%$. From the figures, changing internuclear distance C-N leads to the strong influence on the HHG spectra compared to the experimental errors. Thus, the HHG spectra are very sensitive to the variation of the $\mathrm{C}-\mathrm{N}$ internuclear distance.

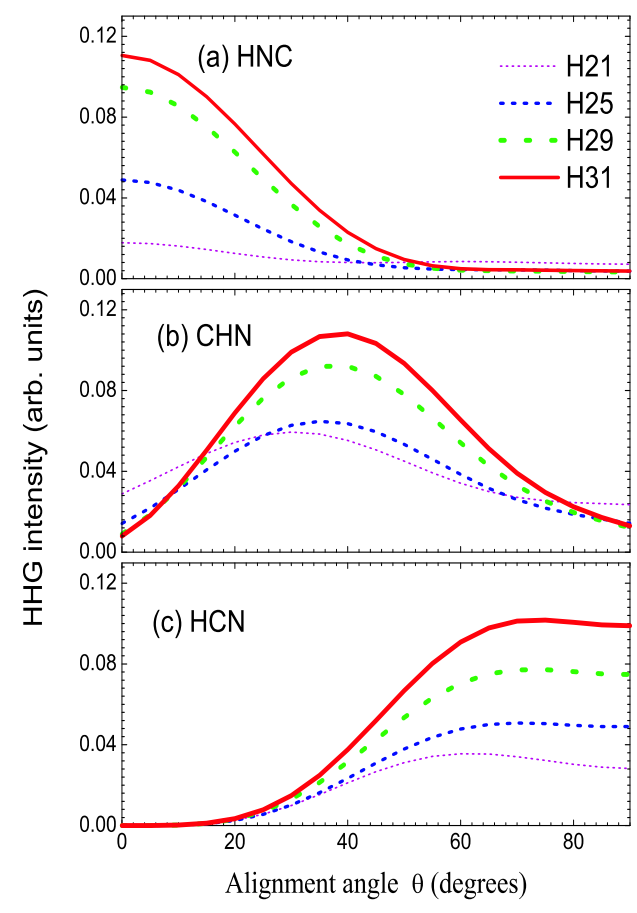

Fig. 1. Alignment dependence of the $21^{\text {st }}, 25^{\text {th }}, 29^{\text {th }}, 31^{\text {st }}$ harmonic yields in plateau region for HCN isomers: (a) hydrogen isocyanide $\mathrm{HNC}$, (b) transition state CHN, and (c) hydrogen cyanide.

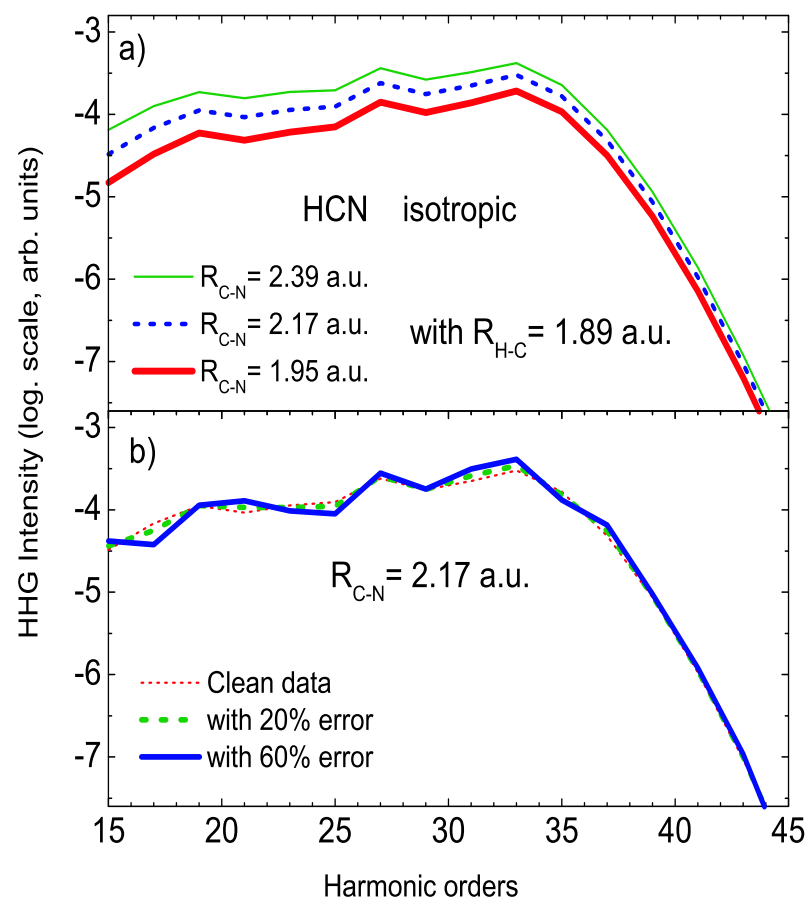

Fig. 2. (a) HHG from HCN with different internuclear distances $\mathrm{C}-\mathrm{N}=2.39$ a.u., 2.17 a.u. and 1.96 a.u. calculated with isotropic alignment (b) HHG from $\mathrm{HCN}$ in equilibrium internuclear distance (R0 $=2.17$ a.u.) with random 'experimental' errors of $20 \%$ and $60 \%$ at each harmonic.

However, contrary to distance $\mathrm{C}-\mathrm{N}$, the influence of bond length $\mathrm{H}-\mathrm{C}$ on the HHG spectra is quite weak. Although the distance H - C decreases or increases by $20 \%$, the HHG spectra change inappreciably. We also obtain the similar results with isomer HNC. It means that the HHG is sensitive to changing the $\mathrm{N}-\mathrm{C}$ distance but not the $\mathrm{H}-\mathrm{N}$ distance.

Following the steps of the iterative method, one can obtain information of the internuclear distance $\mathrm{C}-\mathrm{N}$ from the experimental isotropic HHG for both isomers. The iterative method is unable to apply for the distance $\mathrm{H}-\mathrm{C}$ or $\mathrm{H}-\mathrm{N}$ with error of $10 \%$. However, the motion of $\mathrm{H}$ atom in the molecule, affected by collision with other atoms or the external field, may occur in a large space instead of vibration around the equilibrium position that causes $\mathrm{HCN} / \mathrm{HNC}$ isomerization. In the next sections, we will simulate the isomerization process and show how to track this process by emitted HHG spectra. 


\section{III.2. Simulating the HCN/HNC isomerization}

In Fig. 3, an accurate potential energy surface for the ground doublet electronic state shows the stable state HCN and the meta-stable HNC with difference potential in $0.61 \mathrm{eV}$. The connection of global state and local state is saddled-shape path of barrier's height $2.05 \mathrm{eV}$ describing a transition state at $\theta_{H} \sim 79.6^{0}$.

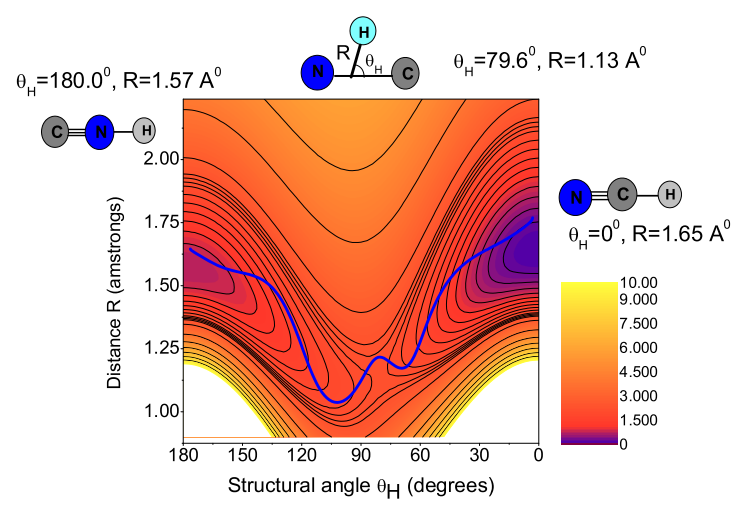

Fig. 3. Potential energy surface (PES) of HCN with corresponding states: hydrogen cyanide, transition state and hydrogen isocyanide. The dark-blue line is a real orbit of $\mathrm{H}$ atom on PES called CRP.

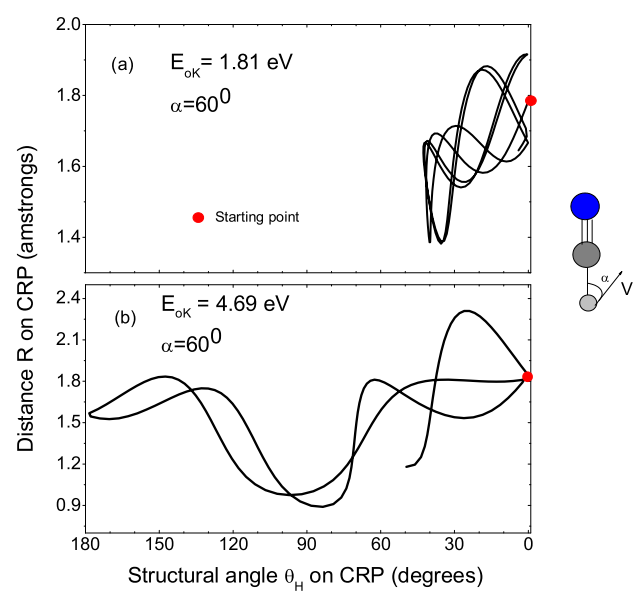

Fig. 4. Orbits of hydrogen atom in space $\left(\mathrm{R}, \theta_{H}\right)$ (a) unfinished isomerization (b) finished isomerization

Direct dynamics study on the lowest doublet potential energy surface has been carried out using electronic structure calculations that follow several classical trajectories with various initial conditions including rotational temperature, velocity of hydrogen atom and its direction. Studies show that the change of rotational temperature from $300 \mathrm{~K}$ even to $5,000 \mathrm{~K}$ does not affect to the process. However, initial kinetic energy and direction of a hydrogen atom are the major causes of isomerization.

Fig. 4a illustrates the orbits of $\mathrm{H}$ atom starting from the stable state $\mathrm{HCN}$ with an initial kinetic energy $E_{0 k}=1.81 \mathrm{eV}$ and moving direction $\alpha=60^{\circ}$. Angle $\alpha$ is between the moving direction of $\mathrm{H}$ atom and the molecular axis. It is obvious that the hydrogen atom cannot pass the potential barrier that result in oscillating around the equilibrium position with the angle amplitude about $\theta_{H} \sim 40^{\circ}$.

Fig. $4 \mathrm{~b}$ shows the case when the initial kinetic energy $E_{0 k}=4.96 \mathrm{eV}$ and the same moving direction $\alpha=60^{\circ}$. In contrary, the hydrogen atom passes the saddle point $(2.16$ $\mathrm{eV}$ ) and HCN transforms to the meta-stable HNC with $\theta_{H} \sim 180^{\circ}$. In Fig. 5, we show the possible area of initial kinetic energy and moving direction where the isomerization process could be happen. 


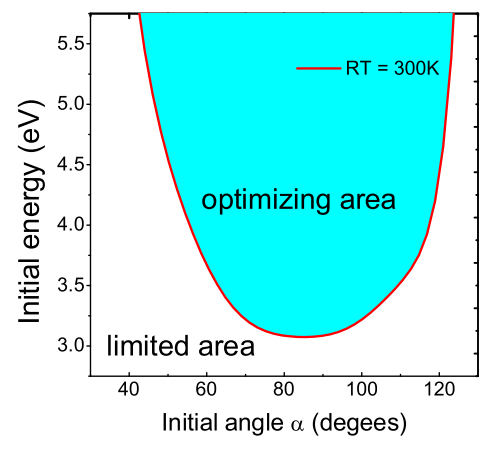

Fig. 5. The region of occurring isomerization (filled)

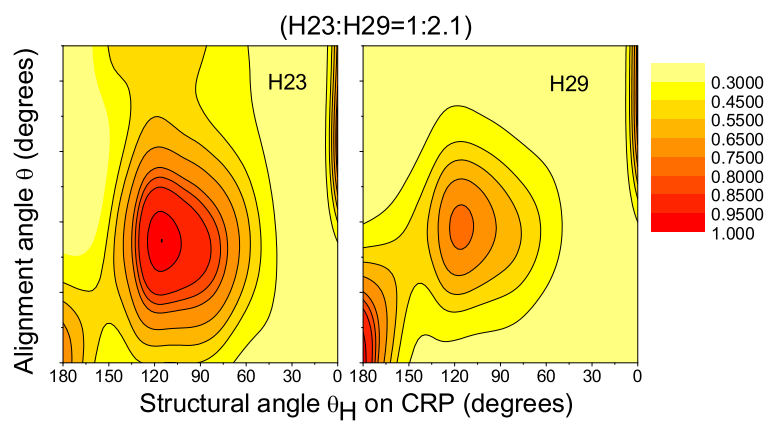

Fig. 6. The dependence of HHG intensity on alignment and position on CRP

\section{III.3. Tracking the isomerization process}

In Fig. 3, we showed one of the saddled-shape orbits of $\mathrm{H}$ atom, called chemical reaction path $(\mathrm{CRP})$ on the potential energy surface. It takes on average $60 \mathrm{fs}$ to transform from $\mathrm{HCN}$ state to HNC state. We calculate the HHG intensity from the molecule during its movement on CRP. Fig. 6 illustrates the dependence of HHG intensity of harmonic orders $23^{r d}$ and $29^{\text {th }}$ on alignments and positions of $\mathrm{H}$ atom on CRP. For any given orbit $(\mathrm{CRP})$, distance $\mathrm{R}$ and structural angle $\theta_{H}$ are dependent. For convenience, we use only angle $\theta_{H}$ to describe positions of $\mathrm{H}$ atom on CRP. In Fig. 6, it is obvious to see that the peaks of HHG intensity occur correspondingly to the isomer positions:HCN $\left(\theta_{H}=0^{0}\right)$, HNC $\left(\theta_{H}=180^{\circ}\right)$ and transition state $\left(\theta_{H} \sim 79.6^{0}\right)$. We can also recheck the results shown in Fig. 1 by analyzing Fig. 6. Indeed, as in Fig. 1 we see in Fig. 6 the HHG peaks at alignments $\theta \sim 0^{0}, \theta \sim 70^{\circ}, \theta \sim 40^{\circ}$ correspondingly for each stable states (HCN and $\mathrm{HNC}$ ) and transition state.

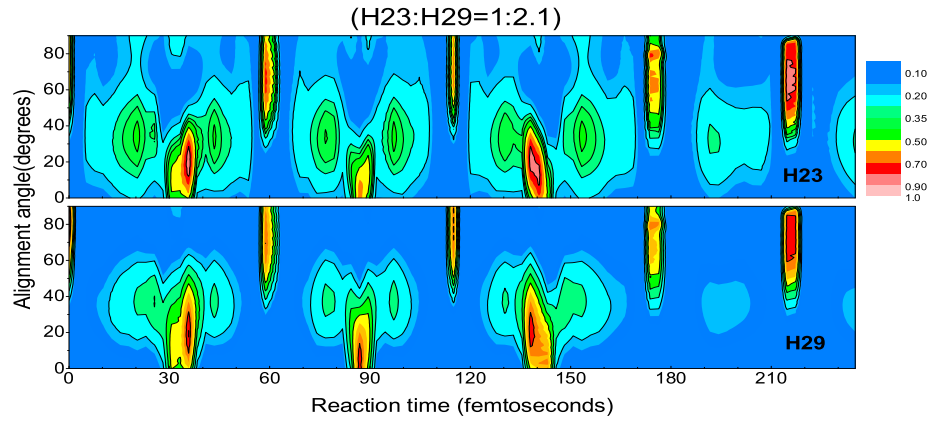

Fig. 7. HHG intensity from HCN during 240fs-process 
Direct dynamics study shows that the molecule will transform from a state to another one until the moving direction and kinetic energy get the values out of the region of isomerization condition as shown in Fig. 5. When the isomerization condition is not satisfied, the molecule will oscillate around stable state, hydro cyanide. The results shown in Fig. 6 are not of practical interest since it is not straightforward to measure $\theta_{H}$ directly in experiment. However, one can observe the isomerizaion process by detecting HHG emitted from the molecule in time domain. In Fig. 7, we illustrate HHG intensity of harmonic orders $23^{r d}$ and $29^{t h}$ dependent on alignments during $240 \mathrm{fs}$ after the isomerization process started. Again, peaks appear clearly at positions of isomers. One can also see that the molecule starts oscillation around stable state after the point of $210 \mathrm{fs}$.

\section{SUMMARY AND OUTLOOK}

In this paper, we have shown how to extract from HHG data the information of isomers HCN. Firstly, from the dependence of the HHG intensity on the molecular alignments we can identify whether isomers are HNC or HCN, even the transition state. Secondly, we have shown the possibility of applying the iterative method to extract the N-C bond length of the molecule from HHG spectra. The change of bond length relating to the $\mathrm{H}$ atom does not affect much on the HHG spectra in order to apply the fitting procedure. However, when the $\mathrm{H}$ atom moves far away from the equilibrium positions such as in the HNC/HCN isomerization process, the HHG spectra may be used to track the isomerization. We simulate the $\mathrm{HNC} / \mathrm{HCN}$ isomerization by using GAUSSIAN with Born-Oppenheimer approximation. By analyzing intensity of HHG, emitted during the isomerization process we see the $\mathrm{HHG}$ peaks nearby the stable $\mathrm{HCN}$, the meta-stable $\mathrm{HNC}$ and the transition state. This may serve as a tool to track the HNC/HCN isomerization process. The physical meaning of the HHG intensity peaks needs to be explained and is under investigation. We will apply further for the other molecules in order to verify whether the existing of HHG intensity peaks at stable states of molecules has the general characters or it is only for the specific case of $\mathrm{HCN} / \mathrm{HNC}$.

\section{REFERENCES}

[1] R. Kienberger et al, Nature 427 (2004) 817.

[2] A. Assion et al, Science 282 (1998) 919.

[3] P. B. Corkum, Phys. Rev. Lett. 71 (1993)1994.

[4] T. Morishita, A. T. Le, Z. Chen, C. D. Lin, Phys. Rev. Lett. 100 (2008) 13903.

[5] S. Minemoto, T. Umegaki, Y. Oguchi, T. Morishita, A. T. Le, S. Watanabe, and H. Sakai, Phys. Rev. A 78, (2008) 061402(R).

[6] R. Torres et al, Phys. Rev. Lett. 98 (2007) 203007.

[7] N. Kajumba et al, New J. Phys. 10 (2008) 025008.

[8] S. Patchkovskii, Z. Zhao, T. Brabec, D. M. Villeneuve, Phys. Rev. Lett. 97 (2006) 123003.

[9] M. Lein, J. Phys. B 40 (2007) R135.

[10] 10. J. Itatani, J. Levesque, D. Zeidler, H. Niikura, H. Pepen, J. C. Kieffer, P. B. Corkum, D. M. Villeneuve, Nature 432 (2004) 867.

[11] V. H. Le, A. T. Le, R. H. Xie, C. D. Lin, Phys. Rev. A 76 (2007) 013414.

[12] V. H. Le, N. T. Nguyen, C. Jin, A. T. Le, C. D. Lin, J. Phys. B 41 (2008) 085603.

[13] M. Lewenstein, Ph. Balcou, M. Yu Ivanov, A. L'Huillier, P. B. Corkum, Phys. Rev. A 49 (1994) 2117. 
[14] A. T. Le, Picca R. Della, P. D. Fainstein, D. A. Telnov, M. Lein and C. D. Lin, J. Phys. B 41 (2008) 081002 .

[15] M. Okunishi, T. Morishita, G. Prumper, K. Shimada, C. D. Lin, S. Watanabe, K. Ueda, Phys. Rev. Lett. 100 (2008) 143001.

[16] R. Bachiller, T. Forveille, P. J. Huggins, P. Cox, Astron. Astrophys. 257 (1992) 701.

[17] R. J. Barber, G. J. Harris, J. Tennyson, J. Chem. Phys. 117 (2002) 11239.

[18] M. Bowman, D. A. Padmavathi, Mol. Phys. 88 (1996) 21.

[19] M. K. Christoffel, M. Bowman, J. Chem. Phys. 112 (2002) 4496.

[20] C. Uiberacker, W. Jakubetz, J. Chem. Phys. 120 (2004) 11532.

[21] I. Vrabel, W. Jakubetz, J. Chem. Phys. 118 (2003) 7366.

[22] M. J. Frisch et al, GAUSSIAN 03, C.02 (2003) (Pittsburgh, PA: Gaussian, Inc.).

[23] K. Takahashi, T. Takayanagi, J. Mol. Struc.: THEOCHEM 817 (2007) 153.

[24] T. Helgaker, E. Uggerud, H. J. A. Jensen, Chem. Phys. Lett. 173 (1990) 145.

Received 17 June 2009. 